Revue internationale P.M.E.

Économie et gestion de la petite et moyenne entreprise

\title{
Conditions et modalités d'émergence des TPE et PE; l'exemple du Maine-et-Loire (France)
}

\section{Alain Capiez}

Volume 1, numéro 2, 1988

URI : https://id.erudit.org/iderudit/1007878ar

DOI : https://doi.org/10.7202/1007878ar

Aller au sommaire du numéro

Éditeur(s)

Presses de l’Université du Québec

ISSN

0776-5436 (imprimé)

1918-9699 (numérique)

Découvrir la revue

Citer cet article

Capiez, A. (1988). Conditions et modalités d'émergence des TPE et PE; l'exemple du Maine-et-Loire (France). Revue internationale P.M.E., 1(2), 127-155.

https://doi.org/10.7202/1007878ar
Résumé de l'article

Cet article présente les résultats d'une enquête sur les TPE émergentes. Quarante-huit entreprises ont répondu à un questionnaire qui a permis un chiffrage ordinal de vingt-quatre variables économiques, organisationnelles, psychologiques et stratégiques. L'analyse de ces variables a permis de déterminer les principales caractéristiques des TPE, d'étudier les liens entre elles, et de mettre en évidence des conditions et des modalités d'émergence : à l'origine de l'émergence, il y a un homme et une stratégie et l'émergence s'effectue en deux stades. 


\title{
Conditions et modalités d'émergence des TPE et PE; l'exemple du Maine-et-Loire (France)
}

\author{
Alain CAPIEZ \\ Université d'Angers
}

\begin{abstract}
RÉSUME
Cet article présente les résultats d'une enquête sur les TPE émergentes. Quarante-huit entreprises ont répondu à un questionnaire qui a permis un chiffrage ordinal de vingt-quatre variables économiques, organisationnelles, psychologiques et stratégiques. L'analyse de ces variables a permis de déterminer les principales caractéristiques des TPE, d'étudier les liens entre elles, et de mettre en évidence des conditions et des modalités d'émergence : à l'origine de l'émergence, il y a un homme et une stratégie et l'émergence s'effectue en deux stades.
\end{abstract}

\begin{abstract}
This article presents the results of an inquiry about rising out $\propto$ Smaller Business Firms" (SBF). Forty-eight firms filled out a questionnaire; the result is an ordinal estimation of twenty-four economical, structural, psychological and strategic variables. The analysis of these variables points out the main characteristics of the SBF, the links between these characteristics, the conditions and ways of rising. The base of rising is a man and a strategy and there are two steps in rising out.
\end{abstract}

\section{RESUMEN}

Este articulo presenta los resultados de una encuesta sobre las empresas muy pequinas (EMP) en fase de surgimento. Cuarenta y ocho empresas respondieron a un cuestionario que ha permitido cifrar veintecuatro variables economicas, de organizacion, sicologicas y estratégicas. Gracias a su analisis, hemos podido determinar las principales caractéristicas de la EMP, estudiar los vinculos que hay entra ellas y poner en evidencia las condiciones en que surgen. Se observa asi que su origin radica a menudo en un hombre y en una estrategia y tiene lugar en dos etapas. 
L'explosion de la création des TPE (toutes petites entreprises) date de la troisième révolution industrielle qui sonne le glas des «Trente Glorieuses». L'objet de cette étude est, à partir d'une enquête portant sur un échantillon de TPE et PE (petites entreprises) émergentes, de préciser les caractéristiques des TPE émergentes et de rechercher les conditions et les modalités de l'émergence des TPE.

Au préalable, il apparaît nécessaire de définir la TPE et la PE; la difficulté d'une définition tient à la grande variété des caractères propres àce type d'entreprise. Diversesétudes ont mis enévidence l'influence personnelle du dirigeant-propriétaire, la propriété des capitaux, le degré d'autonomie décisionnelle, la faiblesse des parts de marché, la structure de l'effectif... Aussi s'accorde-t-on d'abord à privilégier comme indicateur la taille de l'effectif : une TPE a un effectif inférieur à 10 salariés etune PE a un effectif ne dépassant pas 50 salariés. A l'intérieur des TPE, on distingue les «TPE d'adaptation» qui résultent d'une restructuration du système productif en crise et les «TPE d'émergence» qui répondent à un appel du système productif en gestation, cette distinction étant souvent difficile à effectuer, en particulier si l'on tient compte de la stratégie des grandes unités (filialisation, essaimage...). Nous retiendrons la définition de M. Marchesnay (1986), selon laquelle la TPE émergente «résulterait des opportunités technologiques ou commerciales, offertes en termes d'idées ou de produits par la nouvelle donne». Remarquons que la TPE émergente se distingue de l'entreprise artisanale traditionnelle qui «fonctionne normalement sanscroissance, sans investissements réguliers, sans profit notable» (Jaeger, Pouchol et Severs, 1985), que la TPE comprend un nombre restreint de collaborateurs (salariés ou non), que la TPE peut être «à technologie de pointe» mais qu'elle est le plus souvent à technologie moins sophistiquée et répondant à une variété croissante des besoins des particuliers et des entreprises. Ces TPE émergentes, fortement intégrées dans leur micro-environnement local, correspondent au modèle d'hypofirme, proposé pour les seules TPE industrielles; elles sont caractérisées par «des structures peu désintégrées, une recherche de la flexibilité, une aspiration à l'indépendance et à l'intégration socio-économique, au détriment du couple traditionnel profit-croissance» (Marchesnay, 1980). A la différence de l'hyper-firme où la fonction objectif à maximiser est le profit ou la croissance sous la contrainte d'une dimension minimale, dans l'hypo-firme la fonction à maximiser est l'indépendance sous réserve d'une dimension maximale.

Nous définirons l'émergence comme l'obtention d'une masse critique nécessaire pour réaliser la survie et la pérennité de la TPE en affirmant sa compétitivité dans le domaine stratégique visé. La croissance du chiffre d'affaires apparaît alors comme un excellent indicateur d'émergence, mais elle ne constitue pas une fin en elle-même pour la TPE; elle apparaît, dans un premier temps, comme un moyen indispensable à l'existence même de l'entreprise. Si la TPE est parfois considérée comme une entreprise dans l'enfance, c'est un enfant dont la croissance est limitée 
en raison de la priorité accordée par son dirigeant à l'indépendance; la TPE n'est pas destinée à dépasser individuellement une certaine taille, variable selon l'activité et le domaine stratégique, mais qui s'arrêtera, selon toute vraisemblance, à celle de la petite, voire de la «petite-moyenne» entreprise.

Notre approche de l'émergence des TPE comportera trois volets :

- dans le premier, nous préciserons la méthodologie utilisée;

- dans le deuxième, nous présenterons, à partir d'une analyse statistique unidimensionnelle, les aspects des TPE et PE émergentes;

- dans le troisième, nous analyserons les relations entre les variablescaractéristiques des TPE et PE, à l'aide d'une analyse statistique multi-dimensionnelle, afin de rechercher des conditions et des modalités d'émergence; nous verrons ainsi qu'à l'origine de l'émergence des TPE il y a un homme et une stratégie, et que l'émergence peut s'effectuer en deux stades qui montrent le passage de la TPE à la PE.

\section{$2 \quad$ La méthodologie}

Une enquête parquestionnaire aété envoyéeà des PE et des TPE sélectionnées et a donné lieu à un traitement statistique.

\subsection{Le questionnaire}

Le questionnaire a été mis au point après discussion avec des spécialistes concernés par le sujet et testé par interview avec 5 chefs d'entreprise. Le questionnaire définitif, assez important, utilise largement les échelles d'attitude à 5 niveaux qui sont mieux reçues que les questions traditionnelles et permettent des réponses nuancées et mesurables. Il est divisé en 11 parties : identification de l'entreprise, évolution du chiffre d'affaires, composition de l'activité, évolution de la forme juridique de l'entreprise, évolution de l'effectif, évolution de l'organisation de l'entreprise, attitude du dirigeant de l'entreprise, stratégie du développement de l'entreprise, investissements de l'entreprise, importance de l'informatisation de l'entreprise, aide à la création et à l'investissement. Le questionnaire a été envoyé par la poste; les réponses reçues ont été précisées et complétées par entretiens téléphoniques.

\subsection{L'échantillon}

Malgré de récents efforts, les TPE restentencore imparfaitement circonscrites par les statistiques; aussi avons-nous dû recourir à plusieurs organismes consulaires et patronaux pour obtenir un échantillon de 48 entreprises du Maine-et-Loire. Cet 
échantillon est issu d'une population de 350 unités inscrites au répertoire des Métiers de Maine-et-Loire et ayant connu une certaine croissance de leur effectif pendant les 5 dernières années, et de 50 entreprises sélectionnées dans les fichiers des petites entreprises de la CCI d'Angers et du Club des Créateurs d'entreprises d'Anjou et réputées pour leur développement rapide.

Les 48 entreprises retenues se répartissent ainsi, selon le code APE de l'INSEE : 1 dans l'agriculture, 1 dans les industries alimentaires, 11 dans les industries de biens intermédiaires, 7 dans les industries de biens d'équipement, 6 dans les industries de biens de consommation courante, 11 dans les industries de mise en oeuvre du bâtiment, 6 dans le commerce et 6 dans les services. Sur ces 48 entreprises, seules 4 méritent le qualificatif de «firmes à technologie de pointe».

Tab. 1. Caractéristiques générales des TPE

(\% d'entreprises /48)

\begin{tabular}{|c|c|c|c|c|c|}
\hline Classes & 1 & 2 & 3 & 4 & 5 \\
\hline \multirow{2}{*}{ AGE } & $<5$ ans & 5 à 10 ans & $>10$ ans & & \\
\hline & $29,2 \%$ & $54,2 \%$ & $16,6 \%$ & & \\
\hline \multirow{2}{*}{ ORIGINE } & Création & succession & $\begin{array}{l}\text { reprise par } \\
\text { les salariés }\end{array}$ & essaimage & \\
\hline & $56,2 \%$ & $20,8 \%$ & $4,2 \%$ & $18,8 \%$ & \\
\hline \multirow[t]{2}{*}{ FORME JURIDIQUE } & $\begin{array}{l}\text { entreprise } \\
\text { individuelle }\end{array}$ & $\begin{array}{c}\text { société en } \\
\text { commandite } \\
\text { simple }\end{array}$ & SARL & SA & $\begin{array}{c}\text { société } \\
\text { coopérative }\end{array}$ \\
\hline & $10,4 \%$ & $2,1 \%$ & $58,3 \%$ & $22,9 \%$ & $6,3 \%$ \\
\hline \multirow{2}{*}{ ACTIVITÉS } & $\begin{array}{l}1 \text { seule } \\
\text { activité }\end{array}$ & $\begin{array}{c}2 \text { à } 3 \\
\text { activités }\end{array}$ & $\begin{array}{l}4 \text { à } 6 \\
\text { activités }\end{array}$ & & \\
\hline & $45,8 \%$ & $39,6 \%$ & $14,6 \%$ & & \\
\hline \multirow{2}{*}{ STRUCTURE } & entrepreneuriale & fonctionnelle & divisionnelle & & \\
\hline & $35,4 \%$ & $56,3 \%$ & $8,4 \%$ & & \\
\hline \multirow{2}{*}{$\begin{array}{l}\text { EFFECTIF } \\
\text { TOTAL }\end{array}$} & $3-5$ & $6-10$ & $11-20$ & $21-30$ & $30-41$ \\
\hline & $10,4 \%$ & $43,7 \%$ & $29,2 \%$ & $10,4 \%$ & $6,3 \%$ \\
\hline
\end{tabular}


Leurs caractéristiques générales (tablau 1,p. 130) font apparaître une grande diversité : $29 \%$ des entreprises peuvent être considérées comme en phase de démarrage (âge $<5$ ans); $56 \%$ sont des créations pures; $90 \%$ ont adopté la forme juridique de société, dont $58 \%$ celle de SARL typique des entreprises familiales; le nombre d'activités est relativement restreint : seules $15 \%$ des entreprises ont de 4 à 6 activités; en fonction de la taille de l'effectif, on distingue $54 \%$ de TPE et $46 \%$ de $\mathrm{PE} ; 35 \%$ des entreprises ont une structure qualifiée d'entrepreneuriale, en référence à O. Gélinier (1977), dans laquelle le chef d'entreprise résume quasiment à lui seul l'organisation, et $8 \%$ (des TPE) ont choisi dès le départ une structure divisionnelle dans une recherche de diversification des activités ou des produits. Mais toutes ces entreprises se caractérisent par une forte personnalisation et le désir de s'affirmer, tout en préservant une certaine indépendance.

\subsection{Le traitement statistique}

A partir des réponses aux différentes questions, nous avons retenu pour chaque entreprise 24 variables économiques, organisationnelles, psychologiques et stratégiques; nombre de ces variables ont été mesurées par agrégation de scores (participation à la décision, attitudes face à l'environnement et au risque, complexités commerciale et technologique); toutes ont été regroupées en classes ordonnées (à 5 modalités le plus souvent).

Une analyse uni-dimensionnelle de ces variables permettra, dans un premier temps, de préciser les aspects des TPE et PE émergentes.

Dans un deuxième temps, seront étudiés les liens entre les différentes variables afin de déterminer les variables les plus importantes et de mettre en évidence des conditions et des modalités d'émergence. A cet effet, nous procéderons d'abord à des analyses simultanées des variables prises deux à deux : calcul des coefficients de corrélation de rang (de SPEARMAN) entre deux variables ordonnées et test de STUDENT (avec un seuil de signification $\alpha<0,05$ ), pour les variables les plus significatives calcul du CHI-DEUX à partir des modalités ramenées à trois, afin d'éviter les effectifs trop faibles (avec un seuil de signification $\alpha<0,1$ ). Nous croiserons ensuite les modalités des différentes variables, soit 114, dans un tableau de BURT, sur lequel nous réaliserons une analyse factorielle des correspondances multiples à l'aide des logiciels de l'ADDAD. 


\section{Les aspects des TPE et PE émergentes : Analyse uni-dimensionnelle}

\subsection{Caractéristiques économiques}

Nous avons privilégié le taux de croissance du chiffre d'affaires comme indicateur d'émergence. Mais il nous a semblé intéressant de calculer aussi la croissance de l'effectif, dans la mesure où l'on a constaté que, depuis dix ans, les petites entreprises en croissance avaient fortement contribué à l'emploi.

Nous avons demandé les chiffres d'affaires de 1981 à 1986, de sorte que nous disposons de cinq taux de croissance pour les entreprises d'âge 2 (5 à 10 ans) et de un à quatre pour les entreprises d'âge 1 ( $<5$ ans). Tous les taux de croissance annuels du chiffre d'affaires ont été calculés en volume, à l'aide de l'indice des prix à la consommation; pour chaque entreprise, nous avons calculé le taux de croissance moyen, l'écart-type du taux de croissance et le coefficient de risque, rapport de l'écart-type à la moyenne du taux de croissance. Les taux de croissance et les coefficients de risque ont été ensuite regroupés en cinq classes, de même que les créations d'emplois (tableau 2, p. 132).

Tab. 2. Taux de croissance, coefficient de risque et création d'emplois

(\% d'entreprises /48)

\begin{tabular}{|c|c|c|c|c|c|}
\hline Paramètres & 1 & 2 & 3 & 4 & 5 \\
\hline \multirow{3}{*}{$\begin{array}{c}\text { TAUX DE } \\
\text { CROISSANCE } \\
\text { ANNUEL DU } \\
\text { CHIFFRE } \\
\text { D'AFFAIRES }\end{array}$} & Faible & Modéré & Moyen & Fort & Très fort \\
\hline & $1 \% \quad 3 \%$ & $4 \% \quad 9 \%$ & $10 \% \quad 19 \%$ & $20 \% \quad 50 \%$ & $51 \% \quad 274 \%$ \\
\hline & $6,2 \%$ & $16,7 \%$ & $16,7 \%$ & $50,0 \%$ & $10,4 \%$ \\
\hline \multirow{3}{*}{$\begin{array}{c}\text { COEFFICIENT } \\
\text { DU RISQUE }\end{array}$} & Faible & Modéré & Moyen & Fort & Très fort \\
\hline & $0,1 \quad 0,2$ & $0,3 \quad 0,5$ & $0,6 \quad 0,9$ & $1,0 \quad 2,0$ & $2,1 \quad 4,0$ \\
\hline & $4,2 \%$ & $20,8 \%$ & $37,5 \%$ & $22,9 \%$ & $14,6 \%$ \\
\hline \multirow{2}{*}{$\begin{array}{l}\text { CRÉATION } \\
\text { D'EMPLOIS }\end{array}$} & 0 emploi & $1-4$ emplois & 5-10 emplois & 11-19 emplois & 20-24 emplois \\
\hline & $20,8 \%$ & $35,4 \%$ & $25,0 \%$ & $14,6 \%$ & $4,2 \%$ \\
\hline
\end{tabular}


Dans l'ensemble, les performances en matière de croissance sont très satisfaisantes pour l'ensemble de l'échantillon avec une moyenne de $35,4 \%$ annuels, 37 entreprises dépassant les $10 \%$ annuels; ce sont les entreprises récemment créées, en phase de démarrage, qui réalisent la plus forte croissance. Le risque est aussi important pour l'ensemble des entreprises avec un coefficient de risque moyen voisin de 1,36 entreprises ayant un coefficient supérieur à 0,6 . Le nombre d'emplois créés entre 1981 et 1986, depuis l'apparition de l'entreprise, atteint 171 pour les 48 entreprises, le plus grand nombre revenant aux entreprises d'âge 2 (5 à 10 ans) et aux entreprises résultant de création.

\subsection{Caractéristiques psychologiques des dirigeants}

Dans la petite entreprise caractérisée par une forte personnalisation, les décisions sont forcément centralisées autour du ou des quelques dirigeants. Dans notre échantillon, 30 entreprises ont un seul dirigeant, 2 un dirigeant-ouvrier, 2 deux dirigeants-ouvriers, 1 un dirigeant et un dirigeant-ouvrier, 5 deux dirigeants et 6 trois dirigeants; ces dirigeants sont proches de leurs salariés, la plupart participant au travail de production et/ou de bureau dans les entreprises d'effectif inférieur à 30 personnes, de sorte que l'on parle de «management baladeur» (Saporta, 1986), le dirigeant étant plus souvent dans les services et ateliers qu'à son bureau. Il est alors intéressant de connaître leur conception en matière de participation à la prise de décision ainsi que leur attitude vis-à-vis de l'environnement et leur tempérament en matière de risque, car ce sont des variables essentielles qui conditionnent la stratégie de l'entreprise.

En ce qui concerne le caractère participatif de la prise de décision, les réponses aux cinq affirmations se répartissent sur l'ensemble de l'échelle, même si le mode des séries est souvent situé aux extrémités (tableau 3, p. 134).

L'examen des deux premières affirmations montre que, si dans $50 \%$ des cas (beaucoup et totalement) les dirigeants interviennent dans toutes les décisions, ce n'est que dans $37 \%$ des cas qu'ils prennent seuls les décisions de politique générale; laconsultation des collaborateurs est courante dans $54 \%$ des entreprises et l'autonomie laissée aux collaborateurs au sein de leur fonction est grande (beaucoup et totalement) dans $52 \%$ des cas. Enfin, l'efficacité de la prise décision collégiale est reconnue dans $69 \%$ des cas; en ce qui concerne cette dernière affirmation, il est intéressant de rapporter les réponses au nombre de dirigeants. On peut penser que lorsqu'il y a plusieurs dirigeants (souvent associés), la prise de décision a tendance à être collégiale; et, de fait, c'est le cas dans 5 sur 6 des entreprises à trois dirigeants et de 3 sur 5 des entreprises à deux dirigeants; c'est aussi le cas lorsque les dirigeants sont impliqués de manière importante dans le travail de production et se considèrent comme mi-dirigeant, mi-ouvrier. Pour les entreprises à un seul dirigeant, les réponses sont plus variées, avec les deux-tiers en faveur de la prise de décision collégiale; prise de décision collégiale est ici entendue dans le sens de consultation de certains collaborateurs pour les décisions stratégiques essentiellement; plusieurs dirigeants 
Tab. 3. Participation à la décision

(\% d'entreprises /48)

\begin{tabular}{|c|c|c|c|c|c|}
\hline $\mathrm{P}_{\text {Affirmations }}^{\text {Classes }}$ & $\begin{array}{c}1 \\
\text { Pas du tout }\end{array}$ & $\begin{array}{c}2 \\
\text { Un pou }\end{array}$ & $\begin{array}{c}3 \\
\text { Moyen }\end{array}$ & $\begin{array}{c}4 \\
\text { Beaucoup }\end{array}$ & $\begin{array}{c}5 \\
\text { Totalement }\end{array}$ \\
\hline $\begin{array}{l}\text { 1. Vous participez à la prise de toutes } \\
\text { les décisions, même mineures, car } \\
\text { vous estimez que vous devez tout } \\
\text { contrôler. }\end{array}$ & $\begin{array}{c}5 \\
20,8 \%\end{array}$ & $\begin{array}{c}4 \\
10,4 \%\end{array}$ & $\begin{array}{c}3 \\
18,8 \%\end{array}$ & $\begin{array}{c}2 \\
31,3 \%\end{array}$ & $\begin{array}{c}1 \\
18,7 \%\end{array}$ \\
\hline $\begin{array}{l}\text { 2. Vous prenez seul les décisions de } \\
\text { politique générale }\end{array}$ & $\begin{array}{c}5 \\
22,9 \%\end{array}$ & $\begin{array}{c}4 \\
31,3 \%\end{array}$ & $\begin{array}{c}3 \\
8,3 \%\end{array}$ & $\stackrel{2}{2}$ & $\begin{array}{c}1 \\
20,8 \%\end{array}$ \\
\hline $\begin{array}{l}\text { 3. Vous ne prenez les décisions } \\
\text { importantes qu'après avoir consulté } \\
\text { vos collaborateurs } \\
\text { (subordonnés) }\end{array}$ & $\begin{array}{c}1 \\
16,7 \%\end{array}$ & $\begin{array}{c}2 \\
22,9 \%\end{array}$ & $\begin{array}{c}3 \\
6,2 \%\end{array}$ & $\begin{array}{c}4 \\
18,7 \%\end{array}$ & $\begin{array}{c}5 \\
35,5 \%\end{array}$ \\
\hline $\begin{array}{l}\text { 4. Vous laissez vos collaborateurs } \\
\text { (subordonnés) prendre seuls les } \\
\text { décisions concernant leurs fonctions }\end{array}$ & $\begin{array}{c}1 \\
16,7 \%\end{array}$ & $\begin{array}{c}2 \\
12,5 \%\end{array}$ & $\begin{array}{c}3 \\
18,7 \%\end{array}$ & $\begin{array}{c}4 \\
25,0 \%\end{array}$ & $\begin{array}{c}5 \\
27,1 \%\end{array}$ \\
\hline $\begin{array}{l}\text { 5. Pour être efficace, la prise de } \\
\text { décision doit être collégiale. }\end{array}$ & $\begin{array}{c}1 \\
10,4 \%\end{array}$ & $\stackrel{2}{2}$ & $\begin{array}{c}3 \\
8,3 \%\end{array}$ & $\begin{array}{c}4 \\
33,3 \%\end{array}$ & $\begin{array}{c}5 \\
35,5 \%\end{array}$ \\
\hline IMPORTANCE DE LA PARTICIPATION & $\begin{array}{c}\text { Très faible } \\
6,3 \%\end{array}$ & $\begin{array}{l}\text { Faible } \\
12,5 \%\end{array}$ & $\begin{array}{l}\text { Moyenne } \\
35,4 \%\end{array}$ & $\begin{array}{l}\text { Forte } \\
31,2 \%\end{array}$ & $\begin{array}{c}\text { Très forte } \\
14,6 \%\end{array}$ \\
\hline
\end{tabular}

\begin{tabular}{|l|c|c|c|c|c|}
\hline Nombre de dirigeants & $\begin{array}{c}\text { Pas du } \\
\text { tout }\end{array}$ & Un peu & Moyen & Beaucoup & Totalement \\
\hline 3 & 1 & & & 4 & 1 \\
\hline 2 & & 2 & & & 3 \\
\hline 1,5 (1 dirigeant, 1 dirigeant-ouvrier) & & & & & 1 \\
\hline 1 (2 dirigeants-ouvriers) & & & & & 2 \\
\hline 0,5 (1 dirigeant-ouvrier) & & & & & 2 \\
\hline 1 dirigeant & 4 & 4 & 4 & 12 & 8 \\
\hline \hline ENSEMBLE & 5 & 6 & 4 & 16 & 17 \\
\hline
\end{tabular}


répondent «beaucoup» de préférence à «totalement», parce qu'il «faut bien finir par trancher». La moyenne de ces résultats permet de distinguer cinq profils d'entreprises en matière de participation : environ $19 \%$ des entreprises (faible et très faible) recourent peu à la participation lors des prises de décision, contre près de $15 \%$ se considérant comme très fortement participatives et $31 \%$ fortement participatives; il reste $35 \%$ d'entreprises moyennement participatives. Le décalage vers le pôle participatif peut s'expliquer par la proximité des dirigeants et des salariés dans la TPE, le dirigeant possédant une culture souvent très proche de celle de son personnel. Les dimensions réduites de l'entreprise donnent aux relations interpersonnelles une grande importance, ce qui favorise une certaine attitude participative.

La TPE étant centrée essentiellement sur son dirigeant, il est indispensable, pour connaître le caractère de la stratégie, de prendre en compte son attitude face à l'environnement et son tempérament en matière de risque (tableau 4, p. 136).

Les deux séries de réponses concordent fortement: en matière d'attitude visà-vis de l'environnement, le décalage vers le pôle pro-actif est manifeste ( $83 \%$ dans les classes 4 et 5); en matière d'attitude face au risque, le décalage vers «recherche le risque» existe aussi, mais les résultats sont centrés sur les classes 3 et 4 (83\%): les dirigeantsn'hésitent pasà prendre des risques, maisces risques sont soigneusement calculés. Seuls $6,2 \%$ des dirigeants se qualifient de «fonceurs» et de «battants»; si les autres foncent volontiers vers l'avant, cela ne les empêche pas de regarder sur les côtés, ce qui ralentit peut-être l'impulsion mais leur permet de parer les attaques de flanc! Ces résultats confirment les études sur les caractéristiques des entrepreneurs à succès (Brockaus, 1980), sur les créateurs d'entreprise et sur les étudiants en entrepreneuriat (Sexton et Bowman, 1983; Lorrain et Dussault, 1986) qui sont caractérisés par une propension à prendre des risques calculés. Les dirigeants des TPE de notre échantillon apparaissent ainsi plus comme des «stratèges», des preneurs de risque, que comme des «managers-gestionnaires» qui cherchent à appliquer une stratégie en minimisant les risques, si l'on se réfère à la distinction de M. Marchesnay (1986). Ce sont les caractères de la stratégie des TPE que nous allons rechercher maintenant.

\subsection{La stratégie des TPE et PE}

La stratégie des entreprises de notre échantillon sera abordée successivement sous l'angle de ses caractères généraux et de ses composants.

\subsubsection{Les caractères généraux de la stratégie}

Le caractère de la stratégie apparaît essentiellement offensif : plus de $79 \%$ des réponses sont situées dans les classes 3 et 4; conscientes des menaces de l'environnement, les TPE traquent toutes les opportunités afin de réaliser leur but; le faible pourcentage de la classe 5 confirme que la croissance n'est pas un but en ellemême, mais un moyen d'émerger et qu'elle sera limitée parl'objectif d'indépendance (tableau 5, p. 137). 
Tab. 4. Attitudes face à l'environnement et au risque

(\% d'entreprises /48)

\begin{tabular}{|c|c|c|c|c|c|}
\hline Classes & 1 & 2 & 3 & 4 & 5 \\
\hline \multirow[t]{2}{*}{ A } & $\begin{array}{l}\text { Vous réagissez } \\
\text { uniquement aux actions } \\
\text { de la concurrence }\end{array}$ & & & & $\begin{array}{l}\text { Vous précédez toujours } \\
\text { la concurrence par } \\
\text { vos actions }\end{array}$ \\
\hline & $\begin{array}{c}1 \\
0,0 \%\end{array}$ & $\begin{array}{c}2 \\
2,1 \%\end{array}$ & $\begin{array}{c}3 \\
35,4 \%\end{array}$ & $\begin{array}{c}4 \\
50,0 \%\end{array}$ & $\begin{array}{c}5 \\
12,5 \%\end{array}$ \\
\hline \multirow[t]{2}{*}{ B } & $\begin{array}{l}\text { Vous attendez de } \\
\text { connaître les écolutions de } \\
\text { l'environnement pour } \\
\text { vous y adapter }\end{array}$ & & & & $\begin{array}{l}\text { Vous cherchez à } \\
\text { anticiper l'evvolution } \\
\text { de l'environnement }\end{array}$ \\
\hline & $\begin{array}{c}1 \\
2,1 \%\end{array}$ & $\begin{array}{c}2 \\
6,3 \%\end{array}$ & $\begin{array}{c}3 \\
25,0 \%\end{array}$ & $\begin{array}{c}4 \\
45,8 \%\end{array}$ & $\begin{array}{c}5 \\
20,8 \%\end{array}$ \\
\hline \multirow{2}{*}{$\begin{array}{l}\text { ATTITUDE } \\
\text { ENVIRONNEMENT }\end{array}$} & Réactive & & & & Pro-Active \\
\hline & $0,0 \%$ & $6,3 \%$ & $10,4 \%$ & $62,5 \%$ & $20,8 \%$ \\
\hline \multirow{2}{*}{ C } & $\begin{array}{l}\text { Vous recherchez un } \\
\text { résultat moyen en mini- } \\
\text { misant les risques }\end{array}$ & & & & $\begin{array}{c}\text { Vous recherchez un } \\
\text { résultat maximum en } \\
\text { acceptant un risque } \\
\text { élevé }\end{array}$ \\
\hline & $\begin{array}{c}1 \\
4,2 \%\end{array}$ & $\begin{array}{c}2 \\
10,4 \%\end{array}$ & $\begin{array}{c}3 \\
45,8 \%\end{array}$ & $\begin{array}{c}4 \\
31,3 \%\end{array}$ & $\begin{array}{c}5 \\
8,3 \%\end{array}$ \\
\hline \multirow{2}{*}{ D } & $\begin{array}{c}\text { Vous adoptez un compor- } \\
\text { tement calculateur, modéré } \\
\text { prudent }\end{array}$ & & & & $\begin{array}{l}\text { Vous adoptez un } \\
\text { comportement de } \\
\text { «fonceur», de «battant» }\end{array}$ \\
\hline & $\begin{array}{c}1 \\
6,3 \%\end{array}$ & $\begin{array}{c}2 \\
10,4 \%\end{array}$ & $\begin{array}{c}3 \\
37,5 \%\end{array}$ & $\begin{array}{c}4 \\
39,6 \%\end{array}$ & $\begin{array}{c}5 \\
6,2 \%\end{array}$ \\
\hline \multirow{2}{*}{$E$} & $\begin{array}{l}\text { Vous exploitez de } \\
\text { préférence les créneaux } \\
\text { actuels }\end{array}$ & & & & $\begin{array}{c}\text { Vous recherchez } \\
\text { systématiquement les } \\
\text { nouvelles opportunités }\end{array}$ \\
\hline & $\begin{array}{c}1 \\
2,1 \%\end{array}$ & $\begin{array}{c}2 \\
12,5 \%\end{array}$ & $\begin{array}{c}3 \\
16,6 \%\end{array}$ & $\begin{array}{c}4 \\
43,8 \%\end{array}$ & $\begin{array}{c}5 \\
25,0 \%\end{array}$ \\
\hline \multirow{2}{*}{$\begin{array}{l}\text { ATTITUDE } \\
\text { RISQUE }\end{array}$} & Craint le risque & & & & Recherche le risque \\
\hline & $0,0 \%$ & $12,5 \%$ & $33,3 \%$ & $50,0 \%$ & $4,2 \%$ \\
\hline
\end{tabular}


Tab. 5. Caractères généraux de la stratégle

(\% d'entreprises /48)

\begin{tabular}{|c|c|c|c|c|c|}
\hline $\begin{array}{l}\text { Classes } \\
\text { Caractères de } \\
\text { la stratégie }\end{array}$ & 1 & 2 & 3 & 4 & 5 \\
\hline $\begin{array}{l}\text { Les choix fondamentaux en } \\
\text { matière de développement de } \\
\text { l'entreprise sont:menaces }\end{array}$ & $\begin{array}{l}\text { Uniquement une } \\
\text { réponse à des } \\
\text { menaces } \\
0,0 \%\end{array}$ & $8,3 \%$ & $35,4 \%$ & $43,8 \%$ & $\begin{array}{l}\text { Uniquement une } \\
\text { recherche délibérée de } \\
\text { la croissance } \\
12,5 \%\end{array}$ \\
\hline Mode de croissance & $\begin{array}{c}\text { Croissance interne } \\
75,0 \%\end{array}$ & $\begin{array}{c}\text { Croissance externe } \\
\text { horizontale faible } \\
\text { (1 entrepr.) } \\
14,5 \%\end{array}$ & $\begin{array}{c}\text { Croissance externe } \\
\text { verticale faible } \\
\text { (1 entrepr.) } \\
4,2 \%\end{array}$ & $\begin{array}{c}\text { Croissance externe } \\
\text { horizontale forte } \\
\text { (2 entr. et +) } \\
2,1 \%\end{array}$ & $\begin{array}{l}\text { Croissance externe } \\
\text { concentrique ou verticale } \\
\text { forte } \\
4,2 \%\end{array}$ \\
\hline $\begin{array}{l}\text { Importance du développement } \\
\text { international (\% du chiffre } \\
\text { d'affaires à l'exportation) }\end{array}$ & $\begin{array}{c}\mathrm{Nul} \\
85,3 \%\end{array}$ & $\begin{array}{c}1 \% \text { Très faible } \\
4 \% \\
4,2 \%\end{array}$ & $\begin{array}{ccc}8 \% & & 11 \% \\
& \text { Faible } & \\
& 4,2 \% & \end{array}$ & $\begin{array}{c}40 \% \\
\text { Assez important } \\
4,2 \%\end{array}$ & $\begin{array}{c}65 \% \\
\text { Important } \\
2,1 \%\end{array}$ \\
\hline Financement de la croissance & $\begin{array}{c}\text { Autofinancement } \\
\text { uniquement } \\
4,2 \%\end{array}$ & $\begin{array}{l}\text { Endettement modéré } \\
\qquad 35,4 \%\end{array}$ & $\begin{array}{l}\text { Endettement moyen } \\
\qquad 45,8 \%\end{array}$ & $\begin{array}{l}\text { Endettement fort } \\
\qquad 10,4 \%\end{array}$ & $\begin{array}{l}\text { Endettement maximum } \\
\qquad 4,2 \%\end{array}$ \\
\hline
\end{tabular}


Le mode de croissance privilégié est la croissance interne, mais 12 entreprises réalisent une croissance «en grappes», par constitution de filiales ou acquisitions d'entreprises, elles-mêmes très petites, (de 1 à 5 entreprises), à l'image de la grande firme. Le développement international, qui concerne 7 entreprises, peut apparaître comme le prolongement naturel d'une certaine spécialisation, aucune entreprise exportatrice n'ayant plus de trois activités; la petite taille n'est pas un obstacle à l'exportation, l'entreprise réalisant $65 \%$ de son chiffre d'affaires à l'exportation ayant un effectif total de 10 personnes. Enfin, le financement de la croissance par endettement modéré et moyen est typique d'entreprises accordant la priorité à l'indépendance.

\subsubsection{Les composants de la stratégie}

Les composants de la stratégie étudiés ici sont la complexité commerciale, la complexité technologique, la politique de produits, la politique de coopération et la politique d'investissement.

La complexité commerciale, (tableau 6,p. 139), apparaît moyenne (62,5\% de l'effectif en classe 3), ce qui correspond au nombre limité d'activités des entreprises de notre échantillon et à la vocation régionale d'une majorité d'entre elles; pour $64 \%$ des entreprises, le nombre de biens et services offerts ne dépasse pas 30; certes, pour $16 \%$ des entreprises est indiqué un nombre allant de 1000 à 4000 (pour l'une d'entre elles, entreprise commerciale), mais il s'agit vraisemblablement plus de marques et de modèles différents que de produits vraiment distincts.

La complexité technologique, (tableau 7, p. 140), concerne essentiellement l'automaticité, c'est-à-dire le degré de mécanisation et d'automatisation, commun aussi bien aux entreprises commerciales et aux entreprises de services qu'aux entreprises industrielles. Les résultats sont nettement plus partagés que pour la complexité commerciale : autour d'un pôle moyen de $25 \%, 35 \%$ des entreprises considèrent la complexité technologique comme très faible et faible, contre $40 \%$ qui la considèrent comme forte et très forte; au total, la complexité technologique apparait plus affirmée que la complexité commerciale. Il s'agit d'ailleurs souvent moins de technologie de pointe que de technologies plus stabilisées; le souci des TPE est surtout d'assurer une évolution régulière de ses techniques, afin de s'adapter rapidement à l'évolution des besoins de la clientèle et de se préparer éventuellement à des sauts innovateurs plus importants. L'informatisation constitue un élément important de la technologie, distinct de l'automaticité dans les entreprises industrielles. Comme l'on constate généralement un processus cumulatif de l'informatisation dans l'entreprise, le degré d'informati-sation a été mesuré en 5 classes à partir du nombre d'activités informatisées. Dans l'ensemble, il apparaît que les entreprises de notre échantillon commencent seulement à s'informatiser, avec une volonté affirmée de poursuivre l'informatisation en particulier pour les tâches administratives et de gestion. 
Tab. 6. Complexité commerclale

(\% d'entreprises /48)

\begin{tabular}{|c|c|c|c|c|c|}
\hline Paramètres & 1 & 2 & 3 & 4 & 5 \\
\hline \multirow{2}{*}{$\begin{array}{l}\text { Homogénéité } \\
\text { des marchés }\end{array}$} & $\begin{array}{c}\text { Très } \\
\text { homogène }\end{array}$ & & & & $\begin{array}{c}\text { Très } \\
\text { hétérogènes }\end{array}$ \\
\hline & $10,4 \%$ & $27,2 \%$ & $29,1 \%$ & $18,8 \%$ & $14,5 \%$ \\
\hline \multirow{2}{*}{$\begin{array}{l}\text { Nombre de produits } \\
\text { (biens ou services) }\end{array}$} & 12 & 35 & $6 \quad 30$ & $50 \quad 200$ & $1000 \quad 4000$ \\
\hline & $8,4 \%$ & $20,8 \%$ & $35,4 \%$ & $20,8 \%$ & $14,6 \%$ \\
\hline \multirow{2}{*}{$\begin{array}{l}\text { Nombre de régions } \\
\text { ou de pays }\end{array}$} & 1 région & plus. régions & France & $2-5$ pays & 6-20 pays \\
\hline & $43,8 \%$ & $20,8 \%$ & $22,9 \%$ & $8,3 \%$ & $4,2 \%$ \\
\hline \multirow{2}{*}{ Caractère de la clientèle } & Simple & & & & Complexe \\
\hline & $14,8 \%$ & $16,8 \%$ & $13,8 \%$ & $36,6 \%$ & $18,0 \%$ \\
\hline \multirow{2}{*}{ Nombre de concurrents } & 1 & 3 & 6 & 1130 & $50 \quad 100$ \\
\hline & $5,9 \%$ & $31,2 \%$ & $23,5 \%$ & $30,6 \%$ & $8,8 \%$ \\
\hline \multirow{2}{*}{ Complexité commerciale } & Très faible & & & & Très forte \\
\hline & $4,1 \%$ & $16,7 \%$ & $62,5 \%$ & $16,7 \%$ & $0,0 \%$ \\
\hline
\end{tabular}

Caractère de la clientèle

\begin{tabular}{|c|c|c|c|c|c|}
\hline Nombre de clients & $\begin{array}{l}130 \\
21,2 \%\end{array}$ & $\begin{array}{c}31 \quad 100 \\
27,3 \%\end{array}$ & $\begin{array}{c}101 \quad 200 \\
15,2 \%\end{array}$ & $\begin{array}{c}201 \quad 700 \\
27,3 \%\end{array}$ & $\begin{array}{c}1300 \quad 2500 \\
9,0 \%\end{array}$ \\
\hline \multirow{2}{*}{ Types de clients } & Un seul type & & & & $\begin{array}{l}\text { Beaucoup de } \\
\text { types différents }\end{array}$ \\
\hline & $8,3 \%$ & $4,2 \%$ & $16,7 \%$ & $41,7 \%$ & $29,1 \%$ \\
\hline \multirow{2}{*}{ Caractère de la clientèle } & Simple & & & & Complexe \\
\hline & $14,8 \%$ & $16,8 \%$ & $13,8 \%$ & $36,6 \%$ & $18,0 \%$ \\
\hline
\end{tabular}


Tab. 7. Complexité technologique, degré d'informatisation et politique de produits

(\% d'entreprises /48)

\begin{tabular}{|c|c|c|c|c|c|}
\hline Paramètres & 1 & 2 & 3 & 4 & 5 \\
\hline \multirow[t]{2}{*}{ Diversité technologique } & $\begin{array}{l}\text { Produits } \\
\text { utilisant la même } \\
\text { technologie }\end{array}$ & & & & $\begin{array}{l}\text { Produits utilisant } \\
\text { des technologies } \\
\text { très différentes }\end{array}$ \\
\hline & $8,3 \%$ & $37,5 \%$ & $18,7 \%$ & $29,2 \%$ & $6,3 \%$ \\
\hline \multirow[t]{2}{*}{ Type de technologie } & $\begin{array}{l}\text { Technobogie } \\
\text { traditionnelle }\end{array}$ & & & & $\begin{array}{l}\text { Technologie de } \\
\text { pointe, ultra } \\
\text { moderne }\end{array}$ \\
\hline & $10,4 \%$ & $25,0 \%$ & $16,7 \%$ & $39,6 \%$ & $8,3 \%$ \\
\hline \multirow{2}{*}{$\begin{array}{l}\text { COMPLEXITE } \\
\text { TECHNOLOGIQUE }\end{array}$} & Très faible & & & & Très forte \\
\hline & $4,2 \%$ & $31,2 \%$ & $25,0 \%$ & $37,5 \%$ & $2,1 \%$ \\
\hline \multirow{2}{*}{$\begin{array}{l}\text { DEGRÉ D'INFOR- } \\
\text { MATISATION }\end{array}$} & Nul & $\begin{array}{c}\text { Fable } \\
\text { (1-3 activites) }\end{array}$ & $\begin{array}{c}\text { Moyen } \\
\text { (4-6 activités) }\end{array}$ & $\begin{array}{c}\text { Fort } \\
\text { (7-9 activités) }\end{array}$ & $\begin{array}{c}\text { Très fort } \\
\text { (10-12 activités) }\end{array}$ \\
\hline & $25,0 \%$ & $29,2 \%$ & $27,1 \%$ & $10,4 \%$ & $8,3 \%$ \\
\hline \multirow{2}{*}{ Standardisation } & $\begin{array}{c}\text { Satisfaction des } \\
\text { besoins individuels }\end{array}$ & & & & $\begin{array}{l}\text { Grande série avec } \\
\text { des produits } \\
\text { standardisés }\end{array}$ \\
\hline & $39,5 \%$ & $27,1 \%$ & $27,1 \%$ & $4,2 \%$ & $2,1 \%$ \\
\hline \multirow{2}{*}{ Prix-qualité } & $\begin{array}{l}\text { Haute qualité, } \\
\text { prix élevé }\end{array}$ & & & & $\begin{array}{l}\text { Qualité standard, pro- } \\
\text { duit bon marché }\end{array}$ \\
\hline & $12,5 \%$ & $37,5 \%$ & $35,4 \%$ & $10,4 \%$ & $4,2 \%$ \\
\hline $\begin{array}{l}\text { POLTIQUE DE } \\
\text { PRODUITS }\end{array}$ & $25,0 \%$ & $31,3 \%$ & $33,3 \%$ & $8,3 \%$ & $2,1 \%$ \\
\hline
\end{tabular}

La comparaison de la complexité commerciale et de la complexité technologique amène à penser que, si les TPE et PE de notre échantillon choisissent une spécialisation dans un ou quelques segments porteurs (créneaux), elles s'appuient sur une technologie évoluée afin d'offrir à leurs clients des produits de qualité.

En matière de politique de produits, l'opinion des dirigeants des TPE et PE peutêtre résumée en «bon rapport qualité-prix etefficacité de service». La compétitivité de l'entreprise est axée non seulement sur le produit lui-même, mais aussi sur les services inclus dans le produit (fiabilité, commodité, service après-vente, conseils à la clientèle...) dans un interface fournisseur-client. 
Tab. 8. Sous-traitance, coopération, investissements et aides

(\% d'entreprises /48)

\begin{tabular}{|c|c|c|c|c|c|}
\hline Paramètres & 1 & 2 & 3 & 4 & 5 \\
\hline \multirow{2}{*}{$\begin{array}{l}\text { IMPORTANCE DE LA } \\
\text { SOUS-TRAITANCE } \\
\text { (48 entreprises) }\end{array}$} & Nulle & $\begin{array}{l}5 \% \quad 20 \% \\
\text { Modéré }\end{array}$ & $\begin{array}{c}30 \% \quad 50 \% \\
\text { Assez } \\
\text { importante }\end{array}$ & $\begin{array}{c}65 \% \quad 70 \% \\
\text { Forte }\end{array}$ & $\begin{array}{c}100 \% \\
\text { Très forte }\end{array}$ \\
\hline & $56,2 \%$ & $18,7 \%$ & $8,4 \%$ & $4,2 \%$ & $12,5 \%$ \\
\hline \multirow{2}{*}{$\begin{array}{l}\text { TYPE DE SOUS- } \\
\text { TRAITANCE } \\
\text { (21 entreprises) }\end{array}$} & $\begin{array}{c}\text { Capacité } \\
\text { uniquement }\end{array}$ & & & & $\begin{array}{l}\text { Spécialité } \\
\text { uniquement }\end{array}$ \\
\hline & $4,8 \%$ & $9,5 \%$ & $23,8 \%$ & $14,3 \%$ & $47,6 \%$ \\
\hline \multirow[t]{2}{*}{$\begin{array}{l}\text { COOPÉRATION } \\
\text { (48 entreprises) }\end{array}$} & Nulle & Avec PME & $\begin{array}{c}\text { Simple avec } \\
\text { de plus grandes } \\
\text { entreprises } \\
\text { (sous-traitance) }\end{array}$ & $\begin{array}{l}\text { Avec PME et } \\
\text { simple avec } \\
\text { de grandes } \\
\text { entreprises }\end{array}$ & $\begin{array}{c}\text { Avec PME et } \\
\text { paticipative avec } \\
\text { de grandes } \\
\text { entreprises }\end{array}$ \\
\hline & $16,6 \%$ & $31,3 \%$ & $10,4 \%$ & $31,3 \%$ & $10,4 \%$ \\
\hline \multirow{2}{*}{$\begin{array}{l}\text { COMPLEXITÉ DES } \\
\text { INVESTISSEMENTS } \\
\text { (48 entreprises) }\end{array}$} & $\begin{array}{c}\text { Faible } \\
\text { (1-2 motifs) }\end{array}$ & $\begin{array}{l}\text { Assez faible } \\
\text { (3-4 motifs) }\end{array}$ & $\begin{array}{l}\text { Moyenne } \\
\text { (5-6 motifs) }\end{array}$ & $\begin{array}{c}\text { Forte } \\
\text { (7-9 motifs) }\end{array}$ & $\begin{array}{c}\text { Très forte } \\
\text { (10-11 motifs) }\end{array}$ \\
\hline & $18,7 \%$ & $27,1 \%$ & $31,3 \%$ & $18,7 \%$ & $4,2 \%$ \\
\hline \multirow{2}{*}{$\begin{array}{l}\text { DNERSTTÉ DES } \\
\text { AIDES } \\
\text { (48 entreprises) }\end{array}$} & $\begin{array}{c}\text { Nulle } \\
\text { (1-2 aides) }\end{array}$ & $\begin{array}{l}\text { Limitée } \\
\text { (3-4 aides) }\end{array}$ & $\begin{array}{l}\text { Moyenne } \\
\text { (5-6 aides) }\end{array}$ & $\begin{array}{l}\text { Variée } \\
\text { (7 aides) }\end{array}$ & Très variée \\
\hline & $4,2 \%$ & $62,5 \%$ & $18,7 \%$ & $12,5 \%$ & $2,1 \%$ \\
\hline \multirow{2}{*}{$\begin{array}{l}\text { EFFICACITÉ } \\
\text { DES AIDES } \\
\text { (46 entreprises) }\end{array}$} & $\begin{array}{l}\text { Sans grande } \\
\text { influence }\end{array}$ & & & & Fondamentale \\
\hline & $34,8 \%$ & $15,2 \%$ & $21,7 \%$ & $19,6 \%$ & $8,7 \%$ \\
\hline
\end{tabular}

Vingt-et-une entreprises de notre échantillon ont choisi la sous-traitance pour se développer, avec une importance variable (de $5 \%$ à $100 \%$ de leur chiffre d'affaires), et la recherche de la plus grande spécialité possible afin de réduire leur vulnérabilité et de s'assurer le plus possible d'autonomie (tableau 8, p. 141). Mais certaines entreprises ( 22 dont 11 sous-traitants) externalisent elles aussi une part de 
leur activité, assez faible il est vrai (au maximum $10 \%$ de leur chiffre d'affaires), de sorte que des liens complexes s'établissent entre elles; il faut encore y ajouter des liens avec des groupes industriels et des centres de recherche, de sorte que l'on peut parler d'une véritable politique de coopération. Les relations formelles (soustraitance, co-traitance, GIE, participations financières) et informelles (relations professionnelles et amicales, groupements divers) des TPE et PE entre elles et avec de grandes entreprises constituent des réseaux variés à l'origine d'un véritable maillage du tissu économique régional.

\section{Tab. 9. Motifs d'investissement}

(\% d'entreprises 148)

\begin{tabular}{|l|c|}
\hline MOTIFS & $\%$ \\
\hline - Accroître la capacité de production des produits existants & $56,3 \%$ \\
- Améliorer la qualité des produits actuels & $52,1 \%$ \\
- Améliorer les conditions de travail du personnel & $50,0 \%$ \\
- Améliorer la formation du personnel & $47,9 \%$ \\
- Améliorer l'image de marque de l'entreprise & $47,9 \%$ \\
- Renforcer le potentiel commercial de l'entreprise & $43,8 \%$ \\
- Fabriquer des produits nouveaux & $37,5 \%$ \\
- Automatiser le processus de production & $35,4 \%$ \\
- Améliorer le traitement de l'information & $25,0 \%$ \\
- Eréer ou renforcer la recherche et développement & $22,9 \%$ \\
- Eatisfaire une réglementation d'ordre public & $20,8 \%$ \\
- Accroître les débouchés & $12,5 \%$ \\
- Acquérir des sources d'approvisionnement & $6,3 \%$ \\
\hline
\end{tabular}

Pour une majorité des entreprisesétudiées, la complexité des investissements ne dépasse pas un niveau moyen, mais l'examen des motifs d'investissement se révèle intéressant (tableau 9,p. 142): les deux premiers motifs cités sontcaractéristiques d'entreprises en émergence, et les deux suivants sont typiques d'entreprises de petite taille à caractère convivial, dans lesquelles le patron est attentif aux problèmes professionnels de ses employés; en outre beaucoup de motifs correspondent à des investissements immatériels, composants de plus en plus essentiels del'investissement actuel. 
Tab. 10. Aldes à la création d'entreprises et à l'investissement

(\% d'entreprises /48)

\begin{tabular}{|l|c|}
\hline AIDES & $\%$ \\
\hline - Prêts bancaires à taux réduit & $34,8 \%$ \\
- Prime régionale à la création d'entreprise & $32,6 \%$ \\
- Prime de création d'emplois & $32,6 \%$ \\
- Exonération d'impôts sur les bénéfices & $32,6 \%$ \\
- Prêt personnel aux créateurs d'entreprises & $26,1 \%$ \\
- Prêts participatifs simplifiés & $21,7 \%$ \\
- Aide d'une pépinière (Municipalité sous forme d'ateliers-relais) & $15,2 \%$ \\
- Prêts du CEPME & $10,9 \%$ \\
- Aide à l'innovation de l'ANVAR & $8,7 \%$ \\
- Participation d'une SDR & $4,3 \%$ \\
- Crédit-bail à taux réduit (du FIN) & $4,3 \%$ \\
- Amortissements exceptionnels & $4,3 \%$ \\
- Prêts participatifs technologiques & $2,2 \%$ \\
- Subvention d'équipement & $2,2 \%$ \\
- Exonération de taxe professionnelle & $2,2 \%$ \\
\hline
\end{tabular}

Enfin, les aides limitées en diversité sont jugées par les dirigeants de notre échantillon limitées en efficacité, (tableau 10, p. 143).

Après avoir caractérisé le comportement des TPE et PE par un certain nombre de variables, il nous faut maintenant étudier les liaisons entre ces variables à l'aide d'une analyse multi-dimensionnelle.

\section{Conditions et modalités d'émergence des TPE : Analyse multidimensionnelle des variables}

Afin de mettre en évidence les conditions et les modalités d'émergence des TPE, nous procèderons à des analyses simultanées des variables prises deux à deux ainsi qu'à une analyse simultanée des différentes modalités de toutes ces variables par une analyse factorielle des correspondances multiples. 
Tab. 11. Corrélations entre les variables les plus significatives (corrélation de Pearson) et Chi-Deux

\begin{tabular}{|c|c|c|c|c|c|c|c|c|c|c|c|c|c|c|}
\hline & TCR & CEM & $P D C$ & AEN & ARS & $\operatorname{CSA}$ & IDI & $\mathrm{CCM}$ & CTL & DIF & PPR & COP & $\mathrm{CIV}$ & DAI \\
\hline Taux de croissance $=T C R$ & - & & & & & & & & & & & & & \\
\hline Création d'emplois = CEM & $\begin{array}{c}. . . * \\
0,37 \\
\stackrel{0 . *}{*} \\
(13,49)\end{array}$ & - & & & & & & & & & & & & \\
\hline Participation à déc. $=$ PDC & $\begin{array}{c}* \\
0,31 \\
(2,88)\end{array}$ & $-0,16$ & - & & & & & & & & & & & \\
\hline Att. environnement = AEN & $\begin{array}{c}\ldots . . \\
0,76 \\
(21,82)\end{array}$ & $\begin{array}{c}. . . \\
0,42 \\
(4,17)\end{array}$ & 0,11 & - & & & & & & & & & & \\
\hline Att. risque $=$ ARS & $\begin{array}{c}\ldots . * \\
0,71 \\
(41,38)\end{array}$ & $\begin{array}{c}\ddot{*} \\
0,32 \\
(12,97)\end{array}$ & 0,18 & $\begin{array}{c}. . * \\
0,75 \\
(14 * * \\
(14,82)\end{array}$ & - & & & & & & & & & \\
\hline Caractère stratégique = CSA & $\begin{array}{c}\ldots . * * \\
0,64 \\
(15,80)\end{array}$ & 0,16 & 0,07 & $\begin{array}{c}. . . . \\
0,57 \\
(10,71)\end{array}$ & $\begin{array}{c}. . . * \\
0,60 \\
(11,29)\end{array}$ & - & & & & & & & & \\
\hline Imp. đøv. internat. = IDI & 0,18 & 0,23 & $\begin{array}{l}-\ddot{3} \\
-0,32 \\
(2,19)\end{array}$ & 0,13 & $-0,01$ & $\begin{array}{l}\ldots . * \\
0,38 \\
(6,53)\end{array}$ & - & & & & & & & \\
\hline $\begin{array}{l}\text { Complexité commerci. = } \\
\text { CCM }\end{array}$ & $\begin{array}{c}\ldots . . \\
0,40 \\
* \\
(9,00)\end{array}$ & $\begin{array}{c}\ldots . . * \\
0,40 \\
(15,53)\end{array}$ & 0,07 & 0,19 & 0,19 & 0,24 & 0,21 & - & & & & & & \\
\hline
\end{tabular}


Tab. 11. Corrélations entre les variables les plus significatives (corrélation de Pearson) et ChLDeux

\begin{tabular}{|c|c|c|c|c|c|c|c|c|c|c|c|c|c|c|}
\hline & TCR & CEM & PDC & AEN & ARS & $\operatorname{CSA}$ & IDI & $\mathrm{CCM}$ & CTL & DIF & PPR & COP & CIV & DAI \\
\hline Complexité tech. = CTL & \begin{tabular}{c|}
...$*$ \\
0,43 \\
$\ddot{*}$ \\
$(8,95)$
\end{tabular} & $\begin{array}{c}\ldots . . \\
0,58 \\
(21,09)\end{array}$ & 0,13 & $\begin{array}{l}0 . . . \\
0,43 \\
(3,54)\end{array}$ & $\begin{array}{c}* \\
0,33 \\
(4,22)\end{array}$ & 0,14 & 0,04 & $\begin{array}{l}\ldots, . . \\
0,43 \\
(16,39)\end{array}$ & - & & & & & \\
\hline Degré d'information = DIF & $\begin{array}{c}. . . * \\
0,52 \\
\ddot{\ddot{*}} \\
(11,21)\end{array}$ & $\begin{array}{c}\ddot{*} \\
0,29 \\
(7,30)\end{array}$ & $\begin{array}{c}\ddot{*} \\
0,29 \\
(5,22)\end{array}$ & $\begin{array}{c}\ldots . . \\
0,59 \\
\ddot{*} \\
(9,51)\end{array}$ & $\begin{array}{l}\cdots \\
0,42 \\
(5,24)\end{array}$ & $\begin{array}{c}\ddot{*} \\
0,30 \\
(6,29)\end{array}$ & $-0,05$ & $\begin{array}{c}\ddot{*} \\
0,27 \\
(7,66)\end{array}$ & $\begin{array}{c}. . .5 \\
0,53 \\
(15,65)\end{array}$ & - & & & & \\
\hline Politique product. = PPR & $\begin{array}{c}\cdots . . * \\
-0,49 \\
(18,57)\end{array}$ & $\begin{array}{c}\cdots . . \\
-0,39 \\
(15,11)\end{array}$ & $-0,18$ & $\begin{array}{c}\cdots . . . \\
-0,46 \\
(5,30)\end{array}$ & $\begin{array}{c}\cdots . * \\
-0,49 \\
(9,99)\end{array}$ & $-0,18$ & $-0,12$ & $\begin{array}{c}* \\
-0,29 \\
(6,93)\end{array}$ & $\begin{array}{l}\cdots \\
-0,37 \\
(8,82)\end{array}$ & $\begin{array}{c}\ddot{0}, 26 \\
(12,11)\end{array}$ & - & & & \\
\hline Coopération $=$ COP & 0,02 & 0,19 & 0,15 & 0,15 & 0,16 & $-0,11$ & $-0,18$ & $-0,03$ & 0,20 & $-0,08$ & $-0,08$ & - & & \\
\hline $\begin{array}{l}\text { Complexité investis. = } \\
\text { CIV }\end{array}$ & $\begin{array}{c}\ldots . . \\
0,67 \\
(20,03)\end{array}$ & $\begin{array}{l}\cdots . . \\
0,50 \\
(5,80)\end{array}$ & 0,18 & $\begin{array}{c}\ldots . . \\
0,57 \\
(11,11)\end{array}$ & $\begin{array}{l}0,50 \\
0,80\end{array}$ & $\begin{array}{l}\ldots . . \\
0,51 \\
(7,80)\end{array}$ & 0,17 & $\begin{array}{l}\ldots, 3 \\
0,36 \\
(3,61)\end{array}$ & $\begin{array}{c}\ldots . . * \\
0,53 \\
(18,91)\end{array}$ & $\begin{array}{c}. . . \\
0,57 \\
\ddot{0.46}\end{array}$ & $\begin{array}{c}\cdots . * \\
-0,38 \\
(9,56)\end{array}$ & 0,00 & - & \\
\hline Diversité aides $=\mathrm{DAI}$ & $\begin{array}{c}. . . * \\
0,39 \\
(3,67)\end{array}$ & 0,13 & 0,24 & 0,28 & 0,13 & 0,17 & 0,07 & 0,17 & 0,07 & $\begin{array}{c}* \\
0,26 \\
(3,34)\end{array}$ & $-0,22$ & 0,05 & $\begin{array}{c}. . . \\
0,36 \\
(5,77)\end{array}$ & - \\
\hline
\end{tabular}

- significatif pour $\alpha=0,1$

$\because \quad$ significatif pour $\alpha=0,05$

... signiicatif pour $\alpha=0,01$

.... significatif pour $\alpha=0,005$

Les CHIDEUX calcules figurent entre parenthèses; ils somt tous à 4 degrés de liberté. 


\subsection{Les principales relations entre variables : corrélation et tri croisé}

Nous avons déterminé les liaisons les plus significatives entre les variables prises deux à deux par le calcul des coefficients de corrélation de rang et le test de STUDENT, pour ensuite effectuer un tri croisé entre ces variables significatives avec calcul du CHI-DEUX (tableau 11, p. 144-145).

Tout d'abord, taux de croissance et création d'emplois apparaissent liés dans notre échantillon : les deux indicateurs (test de STUDENT et test du CHI-DEUX) convergent; le tri croisé montre les plus forts pourcentages de création d'emplois dans les entreprises à croissance moyenne et forte et l'absence de création d'emplois principalement dans les entreprises à croissance faible.

En ce qui concerne les caractéristiques psychologiques des dirigeants, la participation à la décision est corrélée négativement avec l'âge de l'entreprise et positivement avec le taux de croissance.

Entre participation à la décision et âge de l'entreprise, il existe un coefficient de corrélation négatif $(-0,44)$ significatif avec un risque $<0,005$. Le croisement de ces deux variables fait apparaître que, dans les entreprises jeunes, il n'y a pas de faible participation mais essentiellement une participation forte, et que c'est dans les entreprises les plus anciennes, que l'on rencontre le pourcentage le plus élevé de faible participation; le test du CHI-DEUX confirme cette dépendance. On peut penser que les dirigeants d'entreprises jeunes sont eux-mêmes des personnes jeunes (du moins d'esprit!) qui adoptent plus volontiers une attitude participative, en raison de leur formation et/ou des nouveaux comportements liés à la forte turbulence économique de ces quinze dernières années, alors que les dirigeants d'entreprises plus anciennes restent plus attachés à un certain individualisme.

Entre participation à la décision et taux de croissance, il existe un coefficient de corrélation positif, mais plus faible $(0,31)$, plus risqué $(<0,05)$ et ne présentant aucune corrélation significative avec les autres variables liées au taux de croissance. De fait, le croisement du taux de croissance et de la participation ne montre aucune tendance vraiment marquée et le test du CHI-DEUX conclut à l'indépendance. Il n'est donc pas possible, à partir de notre échantillon, d'affirmer que l'attitude participative favorise la croissance. Qu'en est-il des autres variables psychologiques ?

Comme le laissait pressentir le tri à plat, l'attitude face à l'environnement, l'attitude face au risque et le caractère de la stratégie sont fortement liées entre elles et avec le taux de croissance. C'est donc bien le caractère du dirigeant, sa capacité à apprécier l'environnement dans lequel se situe son entreprise, son goût du risque calculé, son comportement stratégique qui sont à la base du développement de l'entreprise.

La corrélation positive entre le caractère de la stratégie et l'importance du développement international n'est pas véritablement significative, en raison du 
faible nombre des entreprises exportatrices (7); elles sont toutes situées dans les classes 4 et 5 du caractère de la stratégie («surtout» et «uniquement une recherche de la croissance»), avec les deux entreprises faiblement exportatrices en classe 5 et la plus faiblement exportatrice en classe 4. La corrélation négative entre participation à la décision et importance du développement international, elle, n'est pas du tout significative, les septentreprises étant assez dispersées dans lesclasses de participation, avec la plus fortement exportatrice en classe 1 (très faible participation).

En ce qui concerne les composants de la stratégie, la complexité commerciale et la complexité technologique liées entre elles sont nettement corrélées avec le taux de croissance et la création d'emplois. Le degré d'informatisation, liéà la complexité technologique, est fortement corrélé avec le taux de croissance, mais pas avec la création d'emplois; c'est plus le développement de la technologie quel'informatisation seule qui semble créer des emplois. Quant à la politique de production, elle est corrélée négativement avec le taux de croissance et la création d'emplois ainsi qu'avec la complexité technoloqique. Plus l'environnement commercial devient complexe, en raison de la variété croissante des besoins qui entraîne une grande diversité de la clientèle, plus les TPE répondent par des biens et des services appropriés, grâce à des capacités productives adaptées reposant sur une technologie évoluée et une informatisation croissante.

Ce sont les investissements qui permettent une technologie adaptative, indispensable au développement de l'entreprise; la complexité des investissements estainsiassociée positivement avec la complexité technologique, le taux decroissance, les variables psychologiques et stratégiques, et négativement avec la politique de production.

Enfin la diversité des aides est corrélée positivement avec le taux de croissance et la complexité des investissements, mais dans les deux cas, le test du CHI-DEUX est négatif. Il semble donc que l'opinion des dirigeants sur le caractère limité de l'efficacité des aides soit assez juste. Quant à l'obtention des aides, elle est plus liée à la création d'entreprise, à la création d'emplois et à la nature des investissements, qu'à la variété de ces investissements.

\subsection{Analyse factorielle des correspondances multiples entre les variables}

Une analyse factorielle des correspondances multiples entre les 114 modalités des variables étudiées fera ressortir les variables qui discriminent le mieux les TPE et PE de notre échantillon, afin de déterminer des conditions et des modalités d'émergence des TPE. 
Tab. 12. Codes des modalités des variables caractéristiques des TPE

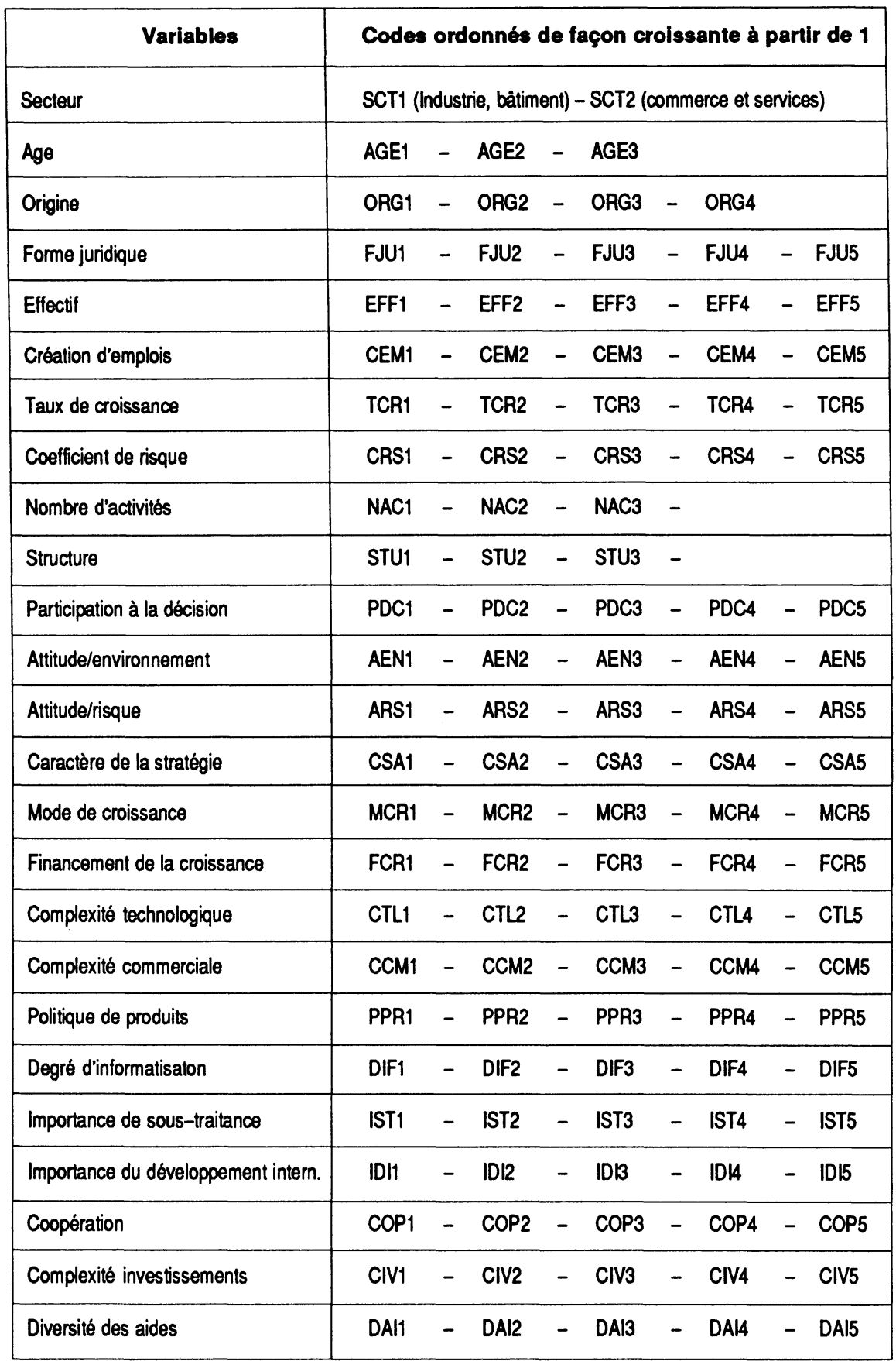


Les quatre premiers axes factoriels ne représentent respectivement que $9,18 \%, 6,24 \%, 5,22 \%$ et $4,77 \%$ de l'inertie du nuage, soit un cumul de $25,40 \%$, en raison de la grande dispersion des données, celle-ci résultant du nombre important de modalités traitées. Cependant la chute d'inertie entre le premier et le second facteur traduit un certain pouvoir structurant du premier axe. Nous analyserons successivement les quatre premiers axes, mais, auparavant, nous examinerons l'évolution des modalités des principales variables dans le plan factoriel $1 \times 2$ (graphique 1 , p. 150).

Les variables les plus contributives de l'axe 1 (TCR, AEN, ARS, CSA) évoluent parallèlement : elles décrivent un croissant, en partant (modalités faibles) de l'extrémité gauche de l'axe 1 en direction de l'extrémité supérieure de l'axe 2, puis en changeant de direction à droite de l'origine pour redescendre brusquement vers l'extrémité inférieure de l'axe 2 (pour les modalités fortes). Seul PPR évolue en sens inverse, de façon moins régulière. Le premier axe factoriel isolera donc les entreprises à faibles performances et le deuxième les entreprises à fortes performances.

\subsubsection{Le premier axe factoriel}

Les modalités les moins contributives se trouvent toutes sur la partie négative de l'axe; ce sont : taux de croissance faible et modéré (TCR1, TCR2), attitude face à l'environnement plutôt réactive (AEN2), attitude face au risque plutôt craintive (ARS2), caractère de la stratégie plutôt défensif (CSA2), politique de produits plutôt standardisés, complexité des investissements faible (CIV1), informatisation nulle (DIF1) et pas de création d'emplois (CEM1).

Cette partie de l'axe fait apparaître les entreprises les moins performantes en raison d'une attitude des dirigeants plutôt réactive et d'une stratégie mal définie; il s'agit des entreprises E16, E9, E13, E1, E46, E35, E39 (graphique 2, p. 151); trois de ces entreprises sont industrielles, trois dans le bâtiment, et une dans les services. L'autre partie de l'axe n'est pas significative.

\subsubsection{Le deuxième axe factoriel}

Comme le montre le graphique d'évolution des modalités des variables, les modalités les plus contributives se trouvent sur la partie négative de l'axe 2; ce sont : taux de croissance très fort (TCR5), attitude pro-active face à l'environnement (AEN5), recherche du risque (ARS5), très forte participation à la décision (PDC5), forte complexité technologique (CTL5), jeunesse (AGE1), faible effectif (EFF1), et, dans une moindre mesure, amorce de développement international (IDI2) et coopération forte (COP5).

Cette partie de l'axe correspond à des entreprises de création récente, à fort taux de croissance en raison d'une stratégie dynamique s'appuyant sur une technologie moderne et évolutive et qui peuvent ébaucher un développement international. Ces caractéristiques pourraient s'appliquer à un premier stade d'émergence des TPE. Les 


\section{Graphique 1 : Plan factoriel 1 × 2 - Les variables}

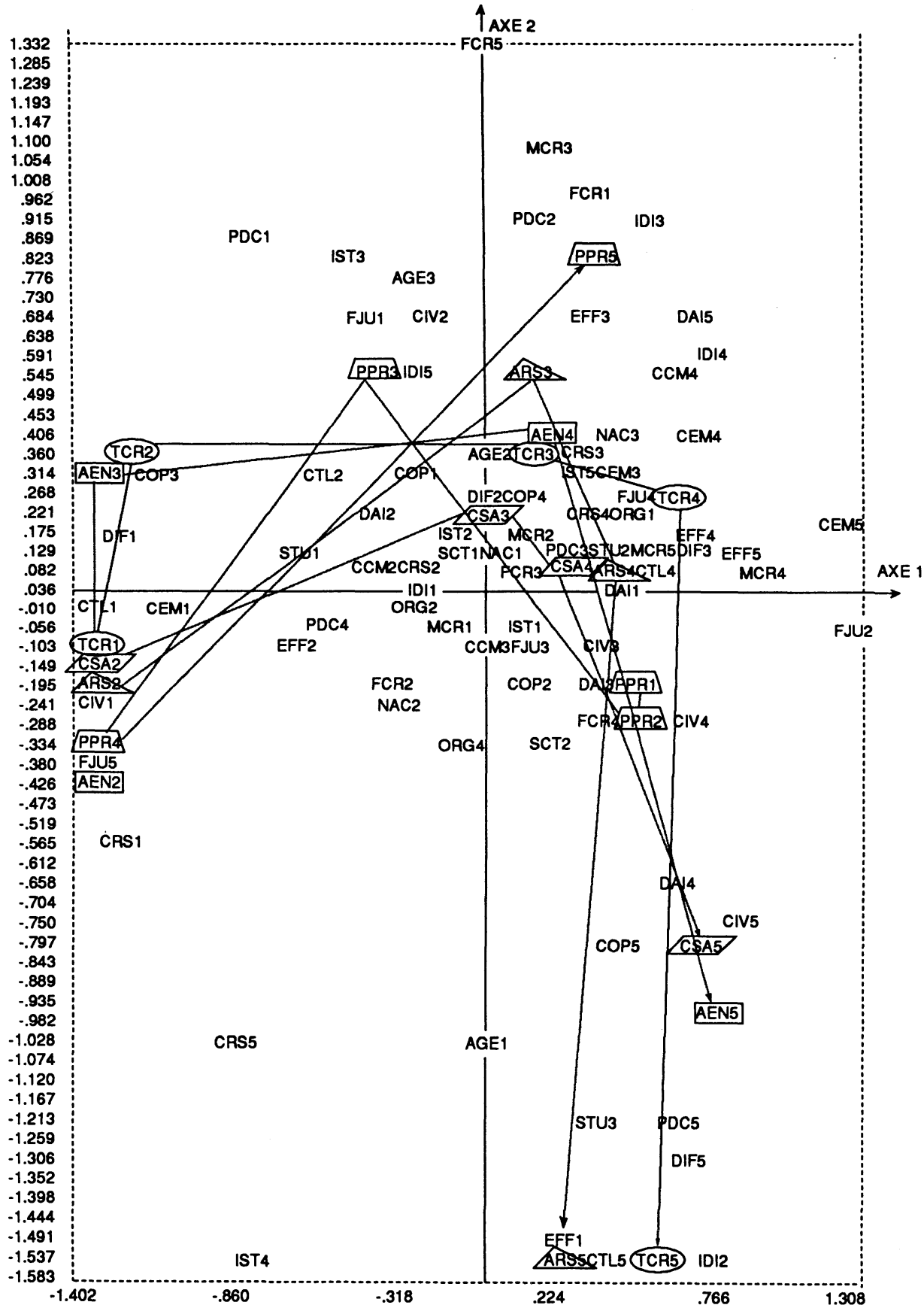




\section{Graphique 2 : Plan factorial $1 \times 2$ - Les entreprises}

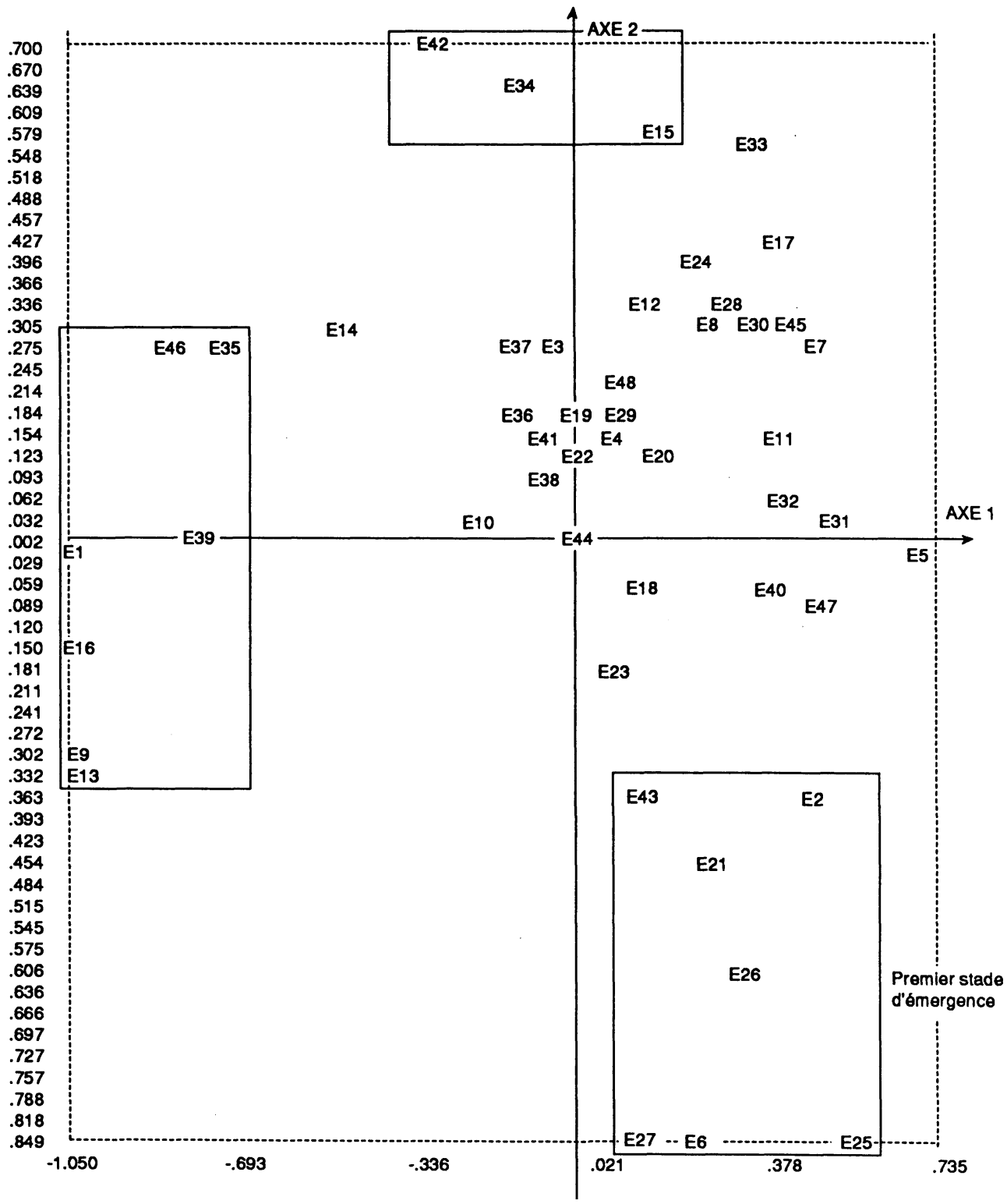


entreprises concernées sont : E6, E27, E25, E26, les deux dernières ayant un développement international très faible; on peut leur rajouter E2, E21, E43; trois de ces entreprises sont industrielles, une dans le bâtiment et trois dans les services.

La partie positive de l'axe 2 regroupe moins de modalités; les plus significatives sont : effectif fort (EFF3), âge plus élevé (AGE2, AGE3), faible participation à la décision (PDC2), fort endettement (FCR5), faible complexité technologique (CTL2). Là, figurent des entreprises plus anciennes, à croissance modérée, à attitude moins participative et à technologie plus traditionnelle; il s'agit des entreprises E42, E34, E15 dont une utilise l'endettement au maximum; ce sont deux entreprises industrielles et une entreprise du bâtiment.

\subsubsection{Le troisième et le quatrième axe factoriel}

Là encore, les modalités les plus contributives figurent sur la partie négative de l'axe 3; ce sont : effectif élevé (EFF5), création d'emplois forte (CEM5), endettement important (FCR4), complexité technologique forte (CTL4), complexité commerciale forte (CCM4) et développement international assez important (IDI4).

Cette partie de l'axe correspond à des entreprises à effectif plus important, à croissance plus stabilisée mais importante, fortement créatrices d'emplois, proposant une gamme plus large de produits, utilisant une technologie plus stable mais évoluée et accentuant l'importance du développement international. Ces caractéristiques pourraient s'appliquerà un deuxième stade d'émergence. Les entreprises concernées sont E5, E4, E11, E47; E5 réalise $40 \%$ de son chiffre d'affaires à l'exportation et E11 se développe par croissance externe forte (MCR5). Il s'agit d'une entreprise agricole, d'une entreprise du bâtiment, d'une entreprise industrielle et d'une entreprise de services. Toutes quatre ont un âge compris entre cinq et dix ans.

La partie positive de l'axe 3 regroupe peu de modalités significatives; les plus contributives sont : effectif moyen (EFF2), création d'emplois faible (CEM2), complexité commerciale moyenne (CCM3), croissance externe faible (MCR2). Il s'agit, ici, d'entreprises à effectif plus faible, moins créatrices d'emplois, mais performantes et se développant plutôt par croissance externe; cette catégorie se recoupe en partie avec celle du premier stade d'émergence: s'y retrouventE26 etE27 entreprises de services appartenant à cette dernière catégorie, et E3 entreprise commerciale.

Sur l'axe 4 figurent peu de modalités significatives; les plus contributives se trouvent sur la partie positive de l'axe; ce sont : financement par endettement maximum (FCR5), développement international modéré (IDI3) et âge important (AGE 3). On trouve ici deux entreprises industrielles créées depuis plus de dix ans, réalisant une forte croissance et qui se sont lancées dans le développement international, E8 et E33, la dernière avec un fort endettement; figure aussi E42, entreprise industrielle très différente des deux autres, avec pour seuls points communs l'âge et l'endettement important comme E33. 
Les axes suivants ne permettent plus aucun regroupement significatif.

L'analyse factorielle fait ainsi apparaître trois catégories d'entreprises : une correspondant aux TPE les moins performantes, les deux autres correspondant à deux stades d'émergence; ces catégories comprennent aussi bien des entreprises industrielles et du bâtiment que des entreprise de services. L'appartenance à l'industrie ou aux services n'a pas d'influence sur les variables étudiées dans notre échantillon; la variable secteur à deux modalités : SCT1 (industrie et bâtiment), SCT2 (commerce et service) n'apparaît à aucun moment significative dans l'analyse factorielle.

\section{Conclusion}

Les conditions d'émergence de la TPE sont un homme et une stratégie.

C'est le dirigeant, le «nouvel entrepreneur» pour employer un terme connu, qui donne son souffle à cette organisation de dimension limitée; en collaboration étroite avec les autres acteurs de son environnement proche, c'est lui qui est à l'origine de l'émergence grâce à sa perception de l'environnement, à sa capacité à prendre des risques quand il décèle des opportunités de développement. En résumé, le dirigeant de TPE est un stratège bien intégré dans l'environnement local.

La stratégie des TPE et PE est limitée car elles doivent s'intégrer dans une carte industrielle complexe dont toutes les grandes zones sont déjà occupées; le dynamisme de leurs dirigeants, l'esprit d'équipe et leur flexibilité les amène à se diriger vers de petits segments porteurs qu'elles vont satisfaire par des biens et des services appropriés, avec des capacités productives adaptées reposant sur une technologie évoluée.

L'émergence des TPE s'effectue selon des modalités qui correspondent à deux stades d'un cycle de vie; certes, il est difficile de généraliser car nombre d'entreprises apparaissent directement à la taille d'une PE, mais les deux stades révélés par l'analyse factorielle constituent un mode de développement possible pour la TPE qui passe progressivement de l'état de TPE à celui de PE.

- Dans un premier stade qui est une phase de démarrage, la TPE récemment apparue, se développe rapidement, réalisant ainsi sa montée en compétitivité, grâce à une stratégie dynamique s'appuyant sur une technologie moderne et très évolutive, afin de suivre et d'anticiper l'évolution des besoins dans le ou les créneaux de marché visés; le dirigeant est alors très participatif; à ce stade, il peut déjà ébaucher un développement international; la coopération avec une grande entreprise peut être une aide appréciable; certaines TPE peuvent déjà se lancer dans la croissance externe.

- Dans un deuxième stade, l'entreprise a atteint une taille plus importante et peut être considérée comme une PE; la croissance se stabilise, mais reste forte et l'entreprise est fortement créatrice d'emplois; la gamme des produits s'élargit et la 
technologie se stabilise tout en restant évoluée; la croissance externe se poursuit pour les entreprises qui ont choisi cette voie; les entreprises qui ont entrepris un développement international l'accentuent. Ce stade correspond plutôt à une phase de croisière, car, selon toute vraisemblance, la TPE ne dépassera pas individuellement une taille-plafond permettant à son dirigeant de rester indépendant.

La TPE réalise ainsi un «apprentissage stratégique», notion d'Igor Ansoff développée par V. de la Ville sous le nom de "gestion stratégique d'apprentissage» (de la Ville, 1988). L'apprentissage stratégique des TPE est fondé sur une coopération avec les acteurs influents de l'environnement local et sur la recherche d'une légitimité. La coopération avec ses pairs et des supérieurs (groupes industriels et centres de recherche) permet à la TPE de mieux cerner les besoins économiques, de se centrer sur des activités permettant d'utiliser au mieux le savoir-faire del'entreprise et de devenir, pour certaines, de véritables partenaires de grandes entreprises. La recherche de l'amélioration de l'image de marque et la volonté de maîtriser des rapports complexes de «lutte-coopération», témoignent d'un souci de légitimité, c'est-à-dire d'une construction de l'identité de la TPE en accord avec le rôle et l'identité des acteurs environnants, réalisant ainsi une véritable symbiose.

En ce qui concerne l'avenir des TPE, on peut penser avec M. Marchesnay (1986) qu'il y a lieu de distinguer les TPE à «technologie de pointe» des autres. Les activités à «technologie de pointe» tendent à se stabiliser progressivement et à être restandardisées; les TPE situées dans ces secteurs seront vraisemblablement absorbées par les grands groupes dont certaines sont issues (essaimage) ou sous la coupe desquelles elles sont déjà par le jeu des contrats d'impartition. Pour les autres, la variété croissante des besoins des usagers, en particulier dans les services, nécessitera un réseau étoffé de TPE et PE, dans une interface fournisseur-client, base essentielle de leur compétitivité.

\section{BIBLIOGRAPHIE}

Albert, Ph., (1986), «Pépinières d'entreprises : un premier diagnostic», Revue Française de Gestion, sept.-octobre, pp. 27-30.

Brockaus, R.H., (1980), «Risk taking propensity of entrepreneurs», Academy of management, vol. 23, $\mathrm{n}^{\circ} 3$, pp. 509-520.

Candau, P., (1981), «Pour une taxonomie de l'hypo-firme», Revue d'économie industrielle, $\mathrm{n}^{\circ} 16$, pp. 16-33.

Debourse, J.P., (1985), «Le comportement exportateur des entreprises artisanales», VIIe journées des LIE, Lille, dans Cahiers de recherche de l'IAE de Lille, $\mathrm{n}^{\circ} 8$.

Gélinier, O.,(1977), L'avenir des entreprisespersonnelles et familiales, Hommes et Techniques, Paris, p. 28.

Huppert, R., (1981), «Stratégies de développement des PMI Françaises», Revue d'économie industrielle, $\mathrm{n}^{\circ} 17, \mathrm{pp} .26-41$. 
Igor Ansoff, H., (1979), «Aspirations and culture in strategic behavior», EIASM working paper, Bruxelles, $\mathrm{n}^{\circ}$ 79-12, pp. 21-25.

Jaeger, C., Pouchol, M. et Severs, M., (1985), «L'artisanat en évolution, l'exemple de trois métiers», Revue d'économie industrielle, n 34, pp. 58-70.

Kakika, M., (1984), Contribution à la connaissance de la structure organisationnelle, essai d'analyse systémique, Thèse d'État, Université de Bordeaux 1.

Lorrain, J., et Dussault, L., (1986), «Les entrepreneurs en phase de démarrage : profil psychologique et comportement de gestion», Revue PMO, vol. 2, n ${ }^{\circ} 1$, pp. 26-36.

Marchesnay, M., (1979), «L'ombre des grands» (en collaboration avec R. Faure et B. Mathie), Revue française de gestion, sept.-octobre, pp. 108-115.

Marchesnay, M., (1980), «Sur la petite entreprise», Revue d' économie industrielle, $\mathrm{n}^{\circ} 11, \mathrm{p}$. 144.

Marchesnay, M., (1982), «Is small so beautiful?», Revue d'économie industrielle, $\mathrm{n}^{\circ} 19, \mathrm{pp}$. 110-114.

Marchesnay, M., (1982) «Pour un modèle d'hypo-firme» dans Entreprises et organisations, Mélanges en l' honneur du professeur J. Aubert-Krier, Economica, Paris, pp. 71-90.

Marchesnay, M., (1986), La stratégie, Du diagnostic à la décision industrielle, Chotard et associés éditeurs, Paris.

Marion, A., «Problématique financière de l'investissement immatériel», Analyse financière, $l^{\text {er }}$ trimestre 1987, pp. 72-82.

Mussault, O. et Perquier, D., (1979), «Cinq stratégies d'entreprises moyennes», Revue Française de Gestion, sept.-oct., pp. 74-80.

Porter, M., (1982), Choix stratégiques et concurrence, Economica, Paris.

Saporta, B., (1986), Stratégies pour la PME, Entreprendre, Montchrestien, Paris.

Sexton, D. et Browman, N., (1983), «Determining entrepreneurial potential of students», Academy of management, proceedings.

Varii Auctores, (1986), La PME en devenir dans un monde en mutation, Actes du colloque international de Trois-Rivières, Presses Universitaires du Québec, en particulier : Fourcade, C., «Le démarrage des entreprises : comparaisons internationales» et Marchesnay, M., «La TPE comme adaptation à la crise».

(de la) Ville, V., (1988), «Comment gérer en situation d'émergence ? Réflexion sur l'intérêt d'une gestion stratégique d'apprentissage», Communication au Colloque International de Montpellier, mai. 